\title{
Falling Suicide Rates in Sri Lanka: lessons and cautions
}

\author{
Diyanath Samarasinghe
}

\section{Summary}

Among the likely major contributors to the recent decline in suicides in Sri Lanka are a reduction in widespread and ready access to highly lethal agrochemicals, better management of persons admitted to hospitals with selfharm and a decline in sensationalized reporting of suicide in print and electronic media - possibly accompanied by a shift in public attitude away from considering self-harm as a natural and expected reaction to any form of distress. Open-minded and concerned discussion should continue, to ensure that gains are improved upon. Attention needs to be paid to some measures that are gradually being eroded.

SL J Psychiatry 2013; 4 (2): 1-3

\section{Introduction}

The very desire to prevent suicides begs the question, 'What is the point of (continuing) life?' Psychiatrists are not professionally qualified to provide an answer. Nor are our somewhat less pretentious siblings, psychologists. Answers we provide to questions such as this are mere individual responses and should not in any way be enhanced by adding an 'expert' veneer. To offer apparent professional opinions regarding matters on which we are not technically required to agree is to descend to the level of astrology and economics.

Although we aren't qualified to demonstrate why attempts at suicide should generally be thwarted, we are expected to give sound scientific advice on how this goal is to be achieved. Our profession may indeed have contributed substantially to the decline in suicides seen in this country across a span of more that a decade now $(1,2)$. In that event, we have reason to be pleased about the soundness of our technical advice. The drop in suicides may on the other hand have happened without any help from us - or even in spite of us. How on earth should we, good scientists, figure out our contribution?

\section{Applying science}

Interpreting varied studies and reports about the alleged underlying causes of suicide is challenging (36). Our prescriptions for preventing suicide can easily be guided by individual whim or preferred worldview. Sound technical opinion, whether on reducing suicide, minimizing alcohol harm or preventing HIV transmission, has to be based on careful and honest weighing of the scientific evidence.

The evidence available restricts us to disappointingly few recommendations. These too are at risk of drawing ridicule because they appear to trivialize the causes of what is probably the gravest act that an individual can contemplate. The public understandably expects to see deep psychological causes being addressed in any preventive actions we recommend. But the strongest evidence-based recommendations for preventing suicides aren't particularly profound or impressive. Nor do most of them give our profession a leading role in implementation.
Reducing ready access to highly lethal agents and stopping or changing media portrayals of suicide, for instance, are not quite convincing or even interesting. To propose minimizing access to strong pesticides (or in the case of the USA, guns) as an appropriate preventive strategy appears the ultimate trivialization of the causes of suicide. The recommendation can be portrayed as an insensitive dismissal of the deep anguish that must prevail for people to contemplate the ultimate act. The public, and the sub-set called 'decision makers', expect to hear proposals such as the provision of strong psychological support and counselling services, effective action to resolve people's life problems and the provision of emergency help for the desperate. The fact that evidence does not support these as effective responses for reducing suicide does not trump the intuitive sense that such proposals make.

Some health sector responses did probably contribute to the improvement we have noted. Among these is the provision of better acute management at the nearest hospital, for agrochemical poisoning. Common sense suggests that effective treatment for several psychiatric disorders that are associated with higher suicide rates - including depression, schizophrenia and dependence on alcohol and other substances - should also help reduce suicides, as will a declining prevalence of alcohol dependence.

We should collectively support continued and enhanced implementation of measures we know to be effective. Many of them require that we stand up to critics who may ridicule some of these responses. Resistance is understandable when people have to face inconveniences in implementing proposed measures, such as making highly lethal agrochemicals less accessible. The same applies to efforts with the mass media to stop the gratuitous, and often sensational, portrayal of suicides. Relentlessly discouraging at least the worst examples of such portrayals is required if we are to win advocates among media personnel too (7). All of these require collective action by the profession. It is easier for a professional body than for individuals to stand up to uninformed (and sometimes informed and malicious) critics. To claim credit for the reduction of suicide in this country, we should first be able to show that we have advocated strongly and collectively for the known effective measures.

Sri Lanka Journal of Psychiatry Vol 4 (2) December 2013 


\section{Critically examining our own beliefs}

We'd do well also to examine our own, often unthinking, assertions. It is understandable that we see a major role for ourselves in the suicide reduction exercise. Our textbooks say that psychiatric disorder is common and severe in those who complete suicide. We too reiterate such opinions. But we also teach that rates of psychiatric disorder are generally similar across countries. How then do we reconcile this with suicide rates differing by more than eight-fold between Greece and Finland, for example? A neat solution is to define suicide itself as a disease (8).

Many of us tend to support the idea that expanding coverage of counselling and specialist psychiatric services will help reduce suicides. A mere glance at statistics from different countries will show that a greater density of psychiatrists is clearly not correlated with lower suicide rates. The trend, if at all, is in the opposite direction. Should any of our detractors demonstrate that there is a significant direct correlation between increased national suicide rates and higher density of psychiatrists, we could of course take refuge in the fact that association does not prove causality.

We could also answer critics by pointing to Sri Lanka, where diminishing suicide rates have been accompanied by increased coverage of psychiatric services. But here too, making a causal connection will not be justified. One mechanism through which improved coverage of psychological services may indeed have helped is through better management of persons with deliberate self harm. This group is particularly important in Sri Lanka firstly because their number is quite large and secondly because they often imbibe highly toxic agrochemicals - unlike 'repeaters' in western countries.

\section{Risk of paradoxical results}

At the same time as we advocate for the known effective measures we should also try to stop well-intentioned responses that carry the potential for making things worse. Measures that make intuitive sense can lead to the opposite of the intended result. Early in the AIDS epidemic some nations shipped victims into camps akin to prisons. The logic appeared sound, but it was abandoned as the impression grew that countries taking this approach were slower in stemming the tide. Whether or not there was a cause and effect relationship there, we should generally bear in mind that unexpected harm can result from well-intentioned actions. Showing scenes on TV of drug users injecting carries a real risk of increasing its prevalence - just as does distributing free disposable syringes to drug users in a low-injecting population. Sincerity of purpose is insufficient guarantee that a given action will not make things worse.

Raising public awareness about the need for more action to prevent suicide may appear to be a good thing to do. So also raising a hue and cry demanding help for people faced with some problem or the other, 'before they commit suicide'. We should be cautious in reinforcing the notion that suicide is an 'automatic' or natural consequence of psychological distress.
This is not to say that we must refrain entirely from advocating for new measures that make intuitive sense. We should press for new responses too. But we have to be alert to the potential for paradoxical outcomes.

\section{Testing new approaches}

Further improving acute management after an act of self harm will probably reduce mortality significantly. A simple measure is to monitor admissions and survival rates after acute poisoning. Variations by district, type of hospital and so on may be revealing. Differences in admissions to bigger hospitals can help spot those in the periphery that do not admit serious cases - simply waving them on, leading to life threatening delays. Measures to improve management of acute poisoning should be applied to facilities where mortality is found to be much higher than the norm. These steps are better taken quietly, than with fanfare that they are intended to prevent suicides.

The dramatic difference in rates between economically similar communities, sometimes almost next door to each other, suggests another avenue to explore. It should be possible, through well-designed interventions, to change the degree to which a given village or community encourages or discourages self harm. Some 'cultures' tend to take self harm lightly, almost as a fact of life or something to be expected when a person is faced with distress. Others may be at the opposite extreme. Such communal views can be made the target of experimental intervention, with careful monitoring of whether public perceptions and acts of self-harm do change.

New threats, currently miniscule, are on the horizon. The 'culture' that encourages suicide is no longer limited to an individual's immediate circle of associates. The effect of belonging to internet-based cultures needs now to be recognized (9-11). Such influences too can be countered, ideally without undue dramatization.

\section{So what do we conclude?}

The recent decline is suicides in Sri Lanka is probably the result of deliberate efforts made to reduce easy access to known highly lethal means of self-harm and to minimize harmful media portrayals. These were likely more effective than in other countries because there was significant public involvement in their implementation. Better management of self-harm likely helped as well. Whether reductions in heavy alcohol use also contributed is unclear. Dramatic declines in response to any intervention can also be explained by the fact that we started from an already horrendously high rate of suicide.

We must ensure that we do not let things slip. Collective, continuous advocacy for even more intensive application of the effective measures is essential. The recent creeping increase of irresponsible media dramatizations of suicide should be countered by united advocacy on our part. We need to mobilize public resistance to these portrayals and not rely solely on action by the 'authorities'. This should come naturally, as we are very much a part of the public. 


\section{Declaration of interest}

None

Diyanath Samarasinghe,

Associate Professor in Psychiatry

Department of Psychological Medicine,

Faculty of Medicine, University of Colombo, Sri Lanka

E mail: hemamali_p@yahoo.com

\section{References}

1. Thalagala N. Suicide Trends in Sri Lanka 1880- 2006: social, demographic and geographical variations. Journal of the College of Community Physicians of Sri Lanka, 2009: 14; 24-32.

2. de Silva V, Hanwella R, Senanayake M. Age and sex specific suicide rates in Sri Lanka from 1995-2011. Sri Lanka Journal of Psychiatry 2012: 3(2); 7-11

3. Editorial. Truth versus myth on mental illness, suicide, and crime. Lancet 2013: 382(9901); 1309.

4. Reardon S. Suicide risk could show up in a blood test. New Scientist 2013: http://www.newscientist.com/ article/dn24070-suicide-risk-could-show-up-in-ablood-test.html\#.UhNEEKyVXxU (accessed Oct 2013)

5. Milner A, Sveticic J, De Leo D. Suicide in the absence of mental disorder? A review of psychological autopsy studies across countries. International Journal of Social Psychiatry 2013: 59 (6); 545-554. http://isp.sagepub. com/content/59/6/545.abstract?etoc (accessed Oct 2013)

6. Germanos A. Downturn Despair: Suicides Spike in Age of Austerity - Report links increase in suicides with 2008 economic crash. http://www.commondreams.org/ headline/2013/09/18-4 (accessed Oct 2013)

7. Foxton W. Reporting suicide: Journalists must resist the temptation to make it a "better story": http://www. newstatesman.com/media/2013/06/reporting-suicidejournalists-must-resist-temptation-make-it-better-story (accessed Oct 2013)

8. Reardon S. Suicidal behaviour is a disease, psychiatrists argue. New Scientist 2013: http://www.newscientist. com/article/dn23566-suicidal-behaviour-is-a-diseasepsychiatrists-argue.html\#.UhNEZKyVXxU (accessed Oct 2013)

9. Ikunaga A, Nath SR, Skinner KA. Internet suicide in Japan: A qualitative content analysis of a suicide bulletin board. Transcultural Psychiatry 2013: 50(2); 280-302. http://tps.sagepub.com/content/50/2/280.abstract?etoc (accessed Oct 2013)

10. Niezen R. Internet suicide: Communities of affirmation and the lethality of communication. Transcultural Psychiatry 2013: 50 (2); 303-322.

11. Kirmayer LJ, Eugene Raikhel E, Rahimi S. Cultures of the Internet: Identity, community and mental health. Transcultural Psychiatry 2013: 50 (2); 165-191. 